\section{Relatório final do VII Seminário de Avaliação dos Cursos de Saúde Pública}

Sérgio Koifman

\section{ANTECEDENTES $(*)$}

As condições criadas pelo processo de discussão com a VIII Conferência Nacional de Saúde tendem a tornar muito particulares as caracter ísticas do próximo Seminário de Avaliação dos Cursos de Saúde Pública.

Embora as linhas centrais que nortearam o encam1nhamento daquele evento há muito viessem sendo discu. tidas no setor saúde - tendo inclusive sido realizadas tentativas de uma operacionalização, num gradiente crescente de complexidade e abrangência - pela primeira vez ocorreu na história das Conferências de Saúde um deba te tão enraizado nos diferentes setores organizados da sociedade civil.

Embora os frutos desse processo de discussão não sejam consensuais - o que seria ingenuidade esperar, uma vez que encontram-se em disputa interesses econômicos poderosos, como os da rede privada de serviços e da indústria de equipamentos no setor saúde - observa-se, por outro lado, uma expressiva legitimidade quan to às principais diretrizes da Conferência: unificação do sistema de saúde e sua progressiva estatização.

Desta maneira, a realização deste VII Seminário de Avaliação dos Cursos de Saúde Pública passa a adquirir uma nova atribuição, qual seja, a de repensar o grau de organicidade que se pretende conferir à formação destes recursos humanos na área de saúde pública, em consonância com uma proposta ratificada por setores expressivos da sociedade.

Em certa medida, as resoluções do VI Semınarıo reaiizado em 1983, na cidade de Nova Friburgo, já tentavam uma aproximação nesta direção. quando a organização dos serviços de saúde foi defin ida como eixo central dos trabalhos em todos os Cursos de Saúde Pública. Assim, a discussão das experiências vivenciadas, desde então, naquele direcionamento, principalmente nos Estados em que o processo de municipalização dos serviços aprofundou-se (São Paulo. Paraná. Minas. Pará, entre outros) representa uma importante fonte de análise do quanto foi possivel avançar em termos de vinculação dos Cursos ao processo iniciado de reestruturação do setor saúde. em geral, e das açóes integradas. em particular.

Contudo, se ocorreran avanços como aqueles acima mencionados, o quadro nacional, quanto à formação de sanitaristas, vem declinando acentuadamente na década de oitenta. culminando com a realização de Cursos convenia-
* Documen to ela horado para leitura dos delegados previamen te à realização do Seminário. 
dos com a ENSP em apenas 5 (cinco) Estados, durante 1985 (Rio Grande do Sul, Paraná, Minas Gerais, Goiás e Pará) além dos Cursos públicos do Estado de São Paulo.

Esta repressão à demanda para formação na área da saúde pública, tem sido um dos principais fatores da proliferação, pelo país, dos Cursos privados de Saúde Pública. Organizados por instituições privadas e realizados em perío. dos noturnos ou durante os finais de semana, estes Cursos têm tido uma grande expansão pelo país, nuito embora não de forma passiva, como demonstra-se através do cancelamento verificado em Pernambuco. O VII Seminário, com a reunião de Coordenadores de Cursos públicos de vá. rios Estados, parece-nos constituir-se no foro ideal para que esta questão, seja analisada e resulte num posicionamento pertinente ao respeito.

Embora a tendência ao declínio esteja em transformação - Rio Grande do Norte, Santa Catarina e Mato Grosso do Sul já iniciaram Cursos este ano, estando previstos para breve, em Alagoas, Pararba, Ceará e Goiás - é preciso garantir a continuidade deste processo.

Para que tal aconteça seria necessário assegurar o caráter permanente dos Cursos, tal como vem ocorrendo há onze anos no Pará e Rio Grande do Sul, sem que estejam à mercê das alterações políticas locais ou à obtenção imedia. tista de resultados. A formação de sanitaristas não pode adquirir o caráter pontual ou intermitente e, para isso, mais do que nunca é necessária a criação ou fortalecimento dos Núcleos de Recursos Humanos ou equivalentes das Instituições de Saúde nos Estados.

Esta diretriz condiciona, por outro lado, um outro desafio, qual seja o de garantir a aproximação e vincula. çẫo com o INAMPS na formação destes recursos.

Até agora, a participação de quadros técnicos da Previdência geralmente vem ocorrendo de forma marginal, secundária. Um projeto de unificação do setor saúde, tal qual tirado como proposta pela VIII Conferência, implica em reposicionamento de todos aqueles envolvidos na forma. ção de recursos humanos, frente aos profissionais daquela Instituição.

Este questionamento necessariamente obriga ao redirecionamento do própric programa dos Cursos, até então concernentes às atividades de caráter ambulatorial e atenção primária. A incorporação dos quadros técnicos da Previdencia e a perspectiva crescente de municipalização, necessariamente nos obrigará à introdução da questão hospitalar, com todas suas implicações no âmbito da atenção secundária e terciária, bem como conteúdos de outra natureza, tais como a vigilância sanitária, proteção ambiental etc.

Uma vez postulados estes parâmetros, que em nosso ponto de vista analisam questões centrais da atual conjuntura dos Cursos de Saúde Pública, tentaremos assinalar 
alguns pontos polêmicos vivenciados pela CONCURD e que este Seminário deverá procurar aprofundar a discussão na busca de respostas.

A proposta de ensino modular, en execução por vários Estados, tem apontado várias deficiências tanto da parte das coordenações como dos próprios alunos, conforme temos tido oportunidade de verificar. Surgida como uma tentativa de eliminar o afastamento prolongado dos alunos em relação aos serviços de saúde, temos observado que, freqüentemente, durante os períodos de dispersão em que aqueles retornam ao serviço, ocorre uma sobrecarga quanto aos trabalhos de rotina, havendo, na prática uma paralisação das atividades relativas ao Curso. Desta maneira, a reavaliação das vantagens e desvantagens deste tipo de programa mereceria ser considerada, procurando-se obter uma recomen dação geral sobre o tema.

A reestruturação do currículo mínimo, em base à análise da experiência de ensino durante os últimos anos, também permitirá rediscutir os conteúdos atuais. Algumas propostas serão apresentadas durante o Seminário, particularmente a da criação de um bloco relacionado à Vigilância Sanitária, além da introdução de conteúdos de Administração Hospitalar, conforme anteriormente salientado.

0 agrupamento dos conteúdos segundo áreas temáticas em substituição às disciplinas individualizadas, também terá oportunidade de ser discutido pela primeira vez desde sua introdução, recomendada em 1983, assim como o próprio ordenamento assumido pelas áreas temáticas, de acordo com a proposta apresentada pelo Grupo de Trabalho, reunido para elaborar o informe daquele Seminário. Neste particular, parece-nos de grande importância comparar as experiências dos Cursos que iniciaram através da área de Saúde e Sociedade, com aqueles que preferiram outros caminhos.

Finalmente, a discussão de proposta atualmente em vigor, da carga horária mínima de 630 (seiscentos e trinta) horas, a duração média de sete a oito meses, as dificuldades em articular o trabalho de campo com reorganização dos serviços da saúde, serão temas também polêmicos, abordados durante o último dia do Seminário.

\section{RELATÓRIO}

\section{Questōes Gerais}

Durante os três dias de discussão no VII Seminário de Avaliação dos Cursos de Saúde Pública, em que pese a exigüidade do tempo, algumas constataçōes puderam ser ratificadas.

Em primeiro lugar, evidenciou-se neste encontro que - acúmulo de experiências durante onze anos de descentralização de Cursos de Saúde Priblica já permitem iniciar os debates com certa compreensão para algumas questões fundamen tais referentes aos mesmos. Assim, tradicionais questionamentos presentes em outros momentos, tais como 
a caracterização do que é a figura do sanitarista, o perfil básico dos conteúdos para sua formação e a relação desta com os serviços de saúde têm respostas que vem sendo consolidadas, em grande parte, ao longo destes onze anos de trabalho conjunto entre as diversas instituiçбes participantes dos Cursos de Saúde Pública.

Um dos obstáculos a esta tarefa tem ocorrido em fun. ção das profundas mudanças verificadas nos núcleos responsáveis pelos Cursos em vários Estados tendo o VII Seminário refletido, em seu desenvolvimento, a heterogeneidade atual das Coordenações Regionais. Contudo, estas diferen. ças não têm sido antagônicas para a continuidade de traba. tho, representando um desafio para encontrar a força e a vitalidade que permitam caminhar juntos, respeitando tais diferenças.

Desta maneira, o Seminário refletiu mais que mudanças de fundo quanto ao redirecionamento dos Cursos de Saúde Pública, uma adaptação que tomasse em consideração aspectos conjunturais da atualidade, mas com profundas repercussões para sua consolidação. Neste sentido, ela pode ser compreendida como uma continuidade do Seminá. rio de Nova Friburgo, cujos objetivos e propostas gerais foram ratificadas, sendo modificadas tão-somente quando necessárias para melhor refletir as transformações desenhadas no país desde 1983 .

Uma das resoluções de maior transcendência do VII Seminário talvez seja a elaboração do documento denominado Car ta do Sumaré (anexo), a qual marca o posicionamen. to dos delegados frente à expansão do ensino privado na área de saúde pública. Um posicionamento firme frente a uma questão de abrangência nacional, e que ocorre de forma coletiva pela primeira vez a partir de setores expressivos da área pública responsável pela formação de recursos humanos, marca certamente uma etapa importante na ampla luta de resgatar o papel do setor público na redemocratização vivida pelo país.

Este posicionamento indica a necessidade não só do aspecto formador, no sentido de designação de respon. sabilidade quanto à formação de sanitaristas no país. mas também pela sua difusão em todos os Estados. multiplicando de forma contínua a formação de quadros técnicos que viabilizem as recomendaçós da VIII Conferencia Nacional de Saúde no que diz respeito a Relorma Samitária.

Reavaliou-se o papel secundário até hoje desempenhado pelo INAMPS neste processo, sendo necessára uma pronta aproximação com aquela instituição, tanto atlavés das coordenaçós locais dos Cursos. como med jante a incorporação substantiva de seus profissiona is na qualidade de alunos.

Fiste tipo de participação, inclusive congregando outras instituçẽes públicas, deve ser operacionalizada através da formação de coleglados multmstituciona is na coordenação dos Cursos de Saúde Pública.

Para que este processo se consolide, o VII Seminário 
ratificou a eleição das Comissões Interestaduais de Saúde como os foros privilegiados para viabilizarem a execução dos Cursos de Saúde Pública. Desta maneira, doravante serão compartilhadas as responsabilidades pela aprovação e financiamento dos mesmos, sem prejuízo no que diz respeito às especificidades regionais para a condução e organização destes Cursos.

Analisou-se também a oportunidade de serem debatidas junto às CIS a importância de constituição, ou reforço quando existen tes, dos Núcleos Estaduais de Formação de Recursos Humanos, com quadro funcional e orçamentário próprios. Em função da própria natureza dos Cursos de Saúde Pública, conforme salientado na Carta do Sumaré, estes não devem caracterizar-se como programas intermitentes, garantindo-se através daquelas propostas os mecanismos para sua continuidade, em condições similares às das açōes básicas de saúde.

Neste aspecto, a proposta de direcionamento dos Cursos de Saúde Pública para a formação de administradores de saúde não foi consensual, uma vez que além de se questionar a viabilização prática dos objetivos de formação globalizante do sanitarista, ela estaria mais adequada enquanto um encaminhamento importante de ser levado a cabo, mas conjuntural e, portanto, em sentido histórico, pontual. Assim, a formação de gerente de saúde, embora considerada oportuna, deveria ser executada como um procedimento incluído na execução dos Cursos de Saúde Pública, simultâneo à formação de sanistaristas já em andamento, e não em substituição a formação daqueles.

Manifestou-se ainda a importância que a ENSP vem mantendo ao longo do processo de descentralização, tanto na qualidade de preservar os Cursos de Saúde Pública como locais sem intervenções de caráter políticopartidário direto, como no sentido de garantir a supervisão acadêmica requerida em distintos níveis pelos Estados. Desta maneira, reafirmou-se a relevância de que esta participação direta da ENSP não só permaneça, como seja ampliada através do repasse de fundos do Ministério da Saúde e Ministério da Previdência e Assistência Social à Escola com o objetivo de fomento específico dos Cursos de Saúde Pública.

Finalmente, foi endossada a proposta vigente de vinculação dos Cursos de Saúde Pública ao fortalecimento das Ações Integradas de Saúde, mesmo tendo-se em conta a grande diversidade entre os Estados. Sublinhou-se, contudo, a importância da manutenção de uma postura reflexiva e crítica e não de subordinação dos Cursos de Saúde Pública aos serviços de saúde, com o que perderiam seu potencial de foro de discussões e ação transformadora sobre aqueles. Neste particular, causa grande preocupação aos participantes a ame aça de retorno à verticalização das ações de saúde, conforme recentemente verificado no controle da epidemia de dengue, em prejuízo não somente da discussão do processo de unificação do setor saúde, mas também enquanto 
retrocesso possível das Açōes Integradas, ainda desigualmente implantadas no país.

\section{Proposta de Ensino}

Em relação à operacionalização dos Cursos de Saúde Pública, o Seminário pronunciou-se pela manutenção da estrutura dos mesmos através das Areas Temáticas preexistentes, sendo sugeridas apenas modificações em seus conteúdos, os quais serão abordados oportunamente.

Para o aperfeiçoamento desta proposta sugeriu-se que ocorra:

a) adequação permanente dos conteúdos temáticos respei tando as especifidades regionais

b) aperfeiçoamento e modernização dos procedimentos didático-pedagógicos do Curso

c) eliminaçẫo das duplicaçōes, min imizaçōes ou superdimensionamentos de temas ou tópicos

d) manutenção de uma equipe local de Coordenação em tempo integral dedicada ao Curso de Saúde Pública, apoiada através de assessoramento pedagógico eventual com cursos de didática para os professores.

Sugeriu-se a manutenção da carga horária atual vigente de 630 horas, procurando-se não acrescentar novas áreas temáticas com vistas à manutenção da unidade e continuidade didático-pedagógica do Curso.

Foi ressaltada a importância de evitar a duplicação de programas verticais nos conteúdos, uma vez que se pretende formar uma postura crítica sobre o funcionamento dos serviços e não apenas o treinamento de profissionais para yiabilizar o funcionamento dos serviços.

$\mathrm{Em}$ realação ao modelo descontinuo de ensino, as discussões durante o Seminário apontaram sua importância mesmo frente aos problemas existentes, considerando-se que em certas circunstâncias (carência de recursos humanos a nivel local, grandes distâncias geográficas etc.), sua adoção é um imperativo; contudo, ressaltou-se a importância de que seja resgatado o sentido inicialmente proposto para os cursos modulares, enquan to programas que analisam uma situação-problema capaz de servir de eixo integrador de diferentes conteúdos e não apenas enquanto cursos descont ínuos no sentido temporal.

Foram salientadas como relevantes as etapas de entre. vistas no processo de seleçăo assim como de avaliação individual durante o Curso. A primeira diz respeito à necessidade de ratificar a avaliação proporcionada pelo exame escrito que, isoladamente, pode mascarar circunstâncias que numa entrevista reorientariam determinados candidatos para opções distintas dos Cursos de Saúde Pública; e quanto à segunda, reflete a necessidade de acompanhamento permanente em diferentes momentos (por exemplo, ao final de cada área temática) do progresso individual dos alunos, sem prejuízo das atividades coletivas de trabalho de campo. 
Em relação aos conteúdos dos Cursos de Saúde Pública, as discussões pelos professores de cada uma das áreas temáticas apontaram para a introdução de algumas modificações em relação à proposta de Nova Friburgo.

A discussão das experiências estaduais ressaltou a importância da área Saúde e Sociedade como introdutória dos Cursos, uma vez que os conteúdos de Ciências Sociais imprimem toda uma dinâmica desejável à abordagem do processo saúde-doença e organização dos serviços de saúde, que não tem sido alcançada em situações distintas.

A área temática de Quantificação dos Problemas de Saúde teve sua denominação modificada para Epidemiologia, embora continue agrupando conteúdos de outras áreas do conhecimento, inclusive ampliando conteúdos de Estatística em relação à proposta de 1983.

A área temática Planejamento e Administração em Saúde teve incorporado um conjunto de conteúdos sobre Administração Hospitalar, que não se constituiu, contudo, em novo bloco para não hipertrofiar o Curso como um todo.

Finalmnte, o conjunto de participantes sugeriu que a Escola Nacional de Saúde Pública (ENSP) realizasse uma investigação de caráter nacional para avaliar a trajetória e situação atual dos egressos dos Cursos de Saúde Pública, como subsídio para a discussão da política de formação de sanitaristas no próximo Seminário Nacional de Coordenadores.

\section{O PONTO DE VISTA DA CONCURD}

Uma vez concluído o Relatório, onde buscou-se exprimir a essência do pensamento coletivamente manifestado durante o Seminário, gostariamos de expressar nossa avaliação particular sobre o momento atual e as perspectivas para os Cursos de Saúde Pública.

Embora os fatores conjunturais tenham sido abordados com relativa abrangên cia (privatização da formação do sanitarista, continuidade dos Cursos, debilidade dos Núcleos de Recursos Humanos, ausência de integração com a Previdência Social nos Estados, vin culação precária dos cursos com as IS, entre outros) faltou, durante aquele encontro, uma análise mais sistematizada sobre o futuro das relações INSP-Cursos Regionalizados.

Con tudo, antes de apontarmos uma proposta de encaminhamen to destas relaçôes, valeria a pena delinear, ainda que em grande pincelada, nossa apreciação sobre a situação nacional dessa modalidade de formação.

Ao longo desta década, em que pese todos os obstáculos mencionados, observou-se a constituição e consolidação de alguns núcleos estaduais com elevada qualificação neste processo formador. A experiência adquirida ao longo de vários anos de organização de Cursos, a preocupação com o detalhamento na sua exe cução (onde a preocupação pela escolha de docentes de disciplinas cedeu lugar

Cadernos de Saúde Pública, $R J ., 3$ (1): 75-80, jan/fov, 1987 
mesmo ao requinte da identificação de pessoas capazes de repassarem certa abordagem em segmentos específicos das mesmas), tornam certas Comissões Coordenadoras altamente gabaritadas, em nada devendo à experiência acumulada na ENSP.

Por outro lado, não é isto, infelizmente, a característica dominante no país,coexistindo, paralelamente, vários Cursos em que muito é preciso avançar. Nestes, pode-se afirmar que não se alcançou ainda uma razoável qualificação dos egressos, entendida como uma formação crítica sobre os determinantes das condições de saúde da população aliada a uma plena capacitação instrumental que possibilite uma prática transformadora nos serviços.

Considerando que, apesar deste diagnóstico a proposta resultante do Seminário foi ampliar a participação do setor público multiplicando os Cursos de Saúde Pública, o que fazer?

Por maior que seja o envolvimento da ENSP neste processo, a ampliação do número de Cursos levará ao esgotamento do atual modelo de atuação, cabendo àquela um mero papel burocrático (seleção, abertura e encerramento de Cursos) em detrimento do seu envolvimento acadêmico na discussão e intercâmbio com os mesmos. Nas atuais condições, a maioria dos Cursos necessitam ser supervisionados e terem analisadas as experiências em desenvolvimento, pois apenas desta maneira estaremos consolidando Núcleos Estaduais que caminharâo a médio prazo para um esta do de relativa au to-suficiência.

Não bastaria supervisão e análise. É necessário investir na capacitação permanente de docentes, por exemplo, na estabilização da infra-estrutura, na coordenação estável etc.

$\mathrm{Na}$ atualidade, a ENSP já não vem conseguindo cum. prir este objetivo, e com a multiplicação dos Cursos, esta situação se agudizará.

Nossa proposta reside na regionalização da atenção às atribuições acadêmicas atualmente cumpridas pela ENSP, sendo estas compartilhadas com alguns Núcleos Estaduais que hoje encontram-se em plenas condiçōes para executarem tais tarefas.

Neste prócesso, caberia à Fundação Oswaldo Cruz o repasse de fundos a cada um destes Núcleos para que se fortaleçam enquanto pólos regionais capacitados financeira e tecnicamente para a execução das atividades de supervisão dos Cursos nas áreas geográficas de sua competência.

Desta maneira, com a criação de três a quatro pólos, estaria assegurada a viabilidade para que estes e a ENSP, em igualdade de atribuiçōes, executassem a contento seu papel no processo de formação de sanitaristas. A CONCURD assim modificada, com representação nacional de fato, teria então plenas condições de transformar a situação vigente, rompendo de vez com os mecanismos de centralização atualmente existentes. 


\section{ÁREA TEMÁTICA l - Saúde e Sociedade}

Carga Horária - 90 horas

Os conteúdos mínimos da Área Temática e Sociedade deverão contemplar aqueles presentes na proposta do "Grupo de Trabalho sobre Avaliação e Proposta para os Cursos de Saúde Públıca - 1984. Friburgo-RJ", acrescidos de fundamentos de Ciências Sociais e de Políticas de Saúde. Desta forma, os conteúdos deverão segur a seguinte sequêencia :

\section{BLOCO 1: Fundamen tos de Ciências Socia is}

a) Correntes do Pensamento em Ciências Sociais.

b) Estudo das categorias em Ciências Sociais: Estado, Ideologia, Classe Social, Luta de Classes, Modo de Produção, Formação Econômica e Social, etc.

c) Processo Histórico do Desenvolvimento Capitalista: - História da Medicinal Social.

d) Desenvolvimento Capitalista na América Latina: - As Práticas Médico-Sanitárias na América Latina.

e) Desenvolvimento Capitalista Brasileiro.

f) Determinação social da doença.

BLOCO II: Políticas de Saúde no Brasil
a) Nacional

b) Regional

\section{BLOCO III : Movimentos Socia is e Saúde Coletiva}

a) Participação popular nas questões da saú de.

b) Partıcipação popular e Estado.

c) A participação popular na gestão dos serviços de saúde.

\section{ÁREA TEMÁTICA II - EPIDEMIOLOGIA}

Carga Horária: 135 horas.

\section{Obje tivos}

1. Adquirir e aplicar métodos e técnicas de diagnóstico e análise das condições de vida c saú de da população e grupos sociais;

2. Conhecer o poten cial e as limitações do sistema de registro de dados e informações dos serviços de saúde;

3. Conhecer è avaliar as ações de vigiância epidemiológica, assim como as políticas de controle.

\section{BLOCO I - Sistemas de Informaçōes em Saúde}

1. Conceitos c usos. A causalidade em saúde. Historicidade do conceito de causa. História natural da docnça (niveis de preven(ְ̧̃̃o). Determ in açũo social da doença.

2. O método estatístico. Fontes de dados primários e secundários. Mítodos da coleta (entrevistas c questionários). Erros do sujeito, do observador e de técnica de observação. Estatísticas vitais. Atestado de óbito. Classificação internacional de doenças.

3. Dados de registro. Util ização de arquivos e manuseio.

4. Dados vitais, cocfecientes a indicadores. Medidas de morbidade, mortalidade e natalidade, Padronizaçāo de coeficientes (método direto).

5. Análise dos dados. Medidas de tendência central e de dispersão. 

e gráfica.

A curva de Gauss, normal e patológico. Apresentação tabu lar

6. Noções de probabilidade. Noçỗes de amostragem. Amostra sistemática e alea tória. Definição e característica.

7. Correlação e regressão.

8. Noçōes de referên cia (teste de diferença entre duas proporções e duas médias).

\section{BLOCO II - Diagnóstico de Saúde} epidem io lógioo.

- Metodologia de investigação. Método científico e método

- Epidemiologia descritiva. Condiçōes de vida e saúde. Indicadores sócioeconômicos, padrões diferenciais de morbi-mortalidade. Variáveis relacionadas a tempo, pessoa e lugar. Variação sazonal.

Tendên cia secular.

- Teoria das epidemias. Determinação social do processo epidêmico. Mensuração de epidemias - diagrama de controle.

- Pirâmide populacional. Estru tura da população brasileira, estimativa do crescimento populacional (método aritmético e geométrico).

Estimativa da populaçẫo brasileira (variaçôes regionais). Urbanização, migraçōes e doença.

- A epidemiologia das doenças transmissíveis e não transmissíveis, incluindo doenças previtáveis por imunizantes. grandes endemias, doenças crônico-degenerativas, doenças carenciais, saúde do trabalhador, acidentes e violências.

\section{BLOCO III - Vigilância Epidem iológica}

- Vigilância epidemiológica. Atribuiçōes. Usos políticos. Democratização da in formação.

- Caracterização de modelos. Políticas su bjacentes.

- Vigilância cpidem iológica das doen ças transmissíveis e não transmissíveis. Vigilância nutricional e de doenças ocupacionais. Inve stigaçōes. Campanhas.

- Políticas de controle. Programas horizontais e verticais.

Política Sanitária.

\section{ÁREA TEMÁTICA III: Ecologia e Saneamento Carga Horária: 90 horas.}

\section{Objetivos}

Evidenciar os conceitos básicos relativos aos sistemas ecológicos e suas modificaçōes, caracterizando a estrutura te dinâmica do ambiente c os principais fatores capazes de alterar o equilibrio destes sistemas bern como os efeitos decorrentes de modificaçôes ambientais sobre o homem.

Conhecer os instrumentos e técnicas u tilizad os para o controle, preservação e recuperação do meio am biente, bem como de sua adequação às diverșs realidades (urband e rural). e estadual.

Analisar a evolução das políticas do setor a nível nacional

\section{Conteú do Programático}

- Conceitos básicos de ecologia

- Iden tificação das variáveis ecológicas no quadro nosológico

- Ambiente e proteção am biental

Cadernos de Saúde Pública, RJ., 3 (1): 75-80, jan $/ \mathrm{fev}, 1987$ 
- Saneamento e saú de

- Princípios gerais de saneamento como instrumento de análise

- Abastecimento e tratamen to de água

- Destino de dejetos e esgotos

- Destino do lixo, coleta, transporte e tratamento

- Controle de vetores e rnedores

- Saneamento em situações emergenciais

- Saneamento am biental: controle da poluição do ar, da água, sonora, visual

- no meio rural

- no meio urbano

- na indústria, etc.

- Inspeção de alimentos

- Vigilância sanjtária

- Atividades de saneamento dos serviços de saúde

- Políticas de saneamento

\section{ÁREA TEMÁTICA VI}

\section{Planeja mento e Administraçâo em Saúde}

Carga Horária: 150 horas.

\section{1 - Introdução ao Panejamento em Saúde}

1. Planejamento, bases conceituais.

O Setor Saúde: como objetivo de planejamento; organização e financiamento do setor. ca Latina;

2. Planejamento em saúde na perspectiva histórica da Améri-

Dar planejamento normativo no planejamento estratégico Análise crítica ao planejamento na América Latina.

3. Planejamen to estratégico

características diferenciais dos enfoques normativo, adaptativo e estratégico - diagnó stico istratégico.

4. Brasil: revisāo histórica e conjuntura sanitária atual.

5. Etapas do planejam ento: construção do plano.

II - Administração Pública e Gerên cia em Serviços de Saúde

1. Determinantes históricoestruturais da burocracia de Estado brasileiro no Brasil.

2. Reformas/modernização de Administração Pública Brasilcira, sistema físcal c orçamentos públicos, financiamento, política de pessoal e apoio logístico do setor saúde.

3. Tcorias Administrativas - fundamen tos básicos.

4. Rede de serviços de saúde e funçōes gerenciais: organização è estrutura; direção e decisão, supervisão e controle, inovação e motivação.

\section{III - Programação}

1. A progamaçāo cono etapa do planejamen to:

- defin içōes, modelos, níveis, ctapas.

- objetivos.

2. Acessibilidade:

- regionalização.

- níveis de complexidade/hicrarquização/conformação da

rede.

3. Diagnóstico Estratégico:

- da população c do estado de saúde.

- da situação epidemiológica.

- diagnóstico da rede/competên cias. 

unidades.

- diagnóstico dos serviços:

- os recursos.

- indicadores de produtividade (inclu indo os hospitalares).

- cadastro para a coleta de informaçōes.

4. Diagnóstico da tecnologia:

- funçōes de produção.

5. Norm atizaçāo:

6. Sistema de informação, avaliação e supervisão:

- metodo logia, níveis e etapas.

7. Sistema de Custos:

8. Adm inistração de Recursos Humanos:

- plano de cargos e salários

- a questão gerencial dos RH

- motivação, participação na gerência e programação das

- controle.

9. Adm inistração de material:

- vantagens e desvantagens da centralização e descentralização.

- as funções: compras/armazenamento

- organização do sistema

- o sistema de material em nível local

10. Adm inis tração Hospitalar:

- o perfil do Hospital na rede

- organização hospita lar

- recursos humanos

- avaliação c controle

- direção hospitalar

11. Reforma Sanitária:

\section{CARGA HORARIA}

Saúde e Socic dade . . . . . . . . . . . . $90 \mathrm{~h}$

Epidem iologia e Quantiticação em Saú de ....... . . $135 \mathrm{~h}$

Ecologia es Sane am ento . . . . . . . . . . $90 \mathrm{~h}$

Plane jamento e Adm inistração dos Serviços de Saúde . $150 \mathrm{~h}$

Atividader Práticas ao longo do curso ......... 165 h

Total . . . . . . . . . . . . . . . . $\overline{630 \mathrm{~h}}$

Relação dos participantes do VII Seminário de Avaliação dos Cursos de Saúde Pública, realizado no Cen tro de Estudos do Sumaré, no período de 02 a 05 de junho de 1986.

01. Conceição Na scimento

Secretaria de Saúde do Estado do Maranhão

Rua Rio Branco, 251

65000 - Sĩo Luís - MA

02. Francisco Emetério Sousa Sales

Secretaria de Saúde do Estado do Cuará

Rua Almirante Barroso, 600 - Praia de Iracema

60000 - Fortaleza - CE.

03. Mário Grassi litho

Faculdade de Ciências da Saúde da

Universidade de Brasûia

70000 - Brasília - Dl:

04. Cairo Alber to de Freitas

Diretoria Regional de Goiânia 
Rua 94 - no 34 - Setor Sul

74000 - Goiânia - GO

05. Cornelis Johannes Van Stralen

Institu to: Cen tro Metropolitano

Rua Atenas, 320, - Ana Lúcia

30000 - Belo Horizonte - MG

06. Carlos Tadeu Villani Marques

Ins titu to: Centro Metropolitano

Rua Alvinópolis, 323 - Apto 301 - Santa Tereza

30000 - Belo Horizonte - MG

07. Túlio Zula to Neto

Instituto: Cen tro Metropolitano

Rua Ma to Grosso, 555/1607 - Barro Preto

30000 - Belo Horizonte - MG

08. Luiz César Nazário Scalia

Cen tro de Ciências Biológicas c da Saú do

Departamento de Medicina

Av. Fernando Correa da Costa, s/no - Caxipó

78000 - Cu jabá - MT

09. Maria Francisca Rangel de Jesus Barros

Fundação Hospitalar do Distrito Federal

Divisāo de Ensino c Aperf eiçoamen to

Coordenadoria do Curso de Saú de Pública

SMHN - Q. $501 \cdot B I . A$

70000 - Brasília - DF

10. Rosely Cerqueira de Oliveira

Secretaria de Suúde do Distrito Federal

Coordenadoria do Curso de Saúde Pública

Ed. Pionciras Socia is, 99 Andar

70000 - Brasília - DF

11. Mascako ly da

Universidade Estadual Paulista

laculdade de Medicina

Departamento de Medicina em Saú de Públical

Rua Tenente Silvio Bestetti. 275

18600 - Botwcatu - SP

12. Isia Maria Hetzel de Maccido

Uni versidade lederal do Rio Grande do Norte

Departamento de Saúde Coletiva e Nutriç̃̃o

Rua Cordeiro de Farias, s/no - Pctrópolis

59000 - Natal - RN

13. Maria de Fátima Saldy

Escola de Saú de Pública de Minas Coerais

Av. Augusto de Lima, 1060

30000 - Belo Horizonte - MCi

14. Ronil da Araújo de Freltas

Ministério da Saúde

Esplanada dos Ministérios - B|. B - 39 And. - S. 347

71600 - Brasilia - Dl:

15. Raimunda Soares Alves

Secretaria de Saúde Pública do Maranhão

Coordenadoria de Recursos Humanos

Rua Rio Branoo, 251

65000 - São Luís - MA

16. Maria Jurandy de Freitas Lóla

C.uernos de Sinude Pública, RJ., 3 (1): 81-97, jan/fev, 1987 
Assessoria de Pós-Graduação

Centro de Ciências da Saúde

Universidade Federal da Para roa

Campus Universitário

58000 - João Pessoa - PB

17. Rosa Maria Quadros Nehmy

Escola de Saúde de Minas Gera is

Coordenadoria de Ensino e Treinamento da ESMIG

Av. Augusto de Lima, 2061

30000 - Belo Horizonte - MG

18. Neide de Queiroz

Universidade Federal de Santa Catarina

Departamento de Saúde Pública

88000 - Florianópolis - SC

19. Elson do Amaral Lima

Secretaria de Saúde de Santa Catarina

Rua Lauro Linhares, $151 / 105$ - Trin dade

88000 - Florianópolis - SC

20. José Geraldo da Costa

Universidade Federal do Maranhão

Caixa Postal, 73

65900 - Imperatriz - MA

21. Marisa Pastori

Faculdade de Medicina de Marília

Rua Azis Atalah, s/nọ - Marília

17500 - Marília - SP

22. Lúcio Spier

Escola de Saúde Pública

Rua D. João VI n 0.73

90000 - Porto Alegre - RS

23. Pio Pereira dos Santos

Secretaria de Saúde do Estado de São Pau lo

UNICAMP

Rua Joāo de Souza Campos, 34

13100 - Campinas - SP

24. Marcos Sales Pimentia

Centro Regional de Saúd.

Av. Andradas, 222

36100 - Juiz de Fora - MG

25. Maria Ruth dos Santos

Rua Mamoré, 36/301 - São Mateus

36100 - Juiz de Fora - MG

26. Maria das Graças Frederico Gomes

Rua Santo Antonio, 786/201 - Centro

36100 - Juiz de Fora - MG

27. Raimunda Isabel Teixeira

Rua Mariano Procópia, 1386/206

36100 - Juiz de Fora - MG

28. Alvaro Gonçalves Neves

Centro Regional de Saú de

Rua Fernando Lobo, 50

36100 - Juiz de Fora - MG

29. Joset ti do Carmo Barbosa de Parada Departamento de Saúde Coletiva 
Institu to de Pa tologia Tropical e Saúde Pública

Universidade Federal de Gojás

Rua 132 n 007 - Setor Sul

74000 - Goiânia - GO

30. Antonio Carlos Porto de Olive ira

Secretaria de Saúde de Rondônia

Esplanada das Secretarias

Av. Farquar

78900 - Porto Velho - RO

31. Lígia Cristina Fonseca Lafoz

Faculdade de Ciências Médicas da Santa Casa

de São Paulo

Departamen to de Medicina Social

Rua Cesário Motta Jr,, 120 - Santa Cecília

05409 -- São Paulo - SP

32. Naila Janilde Seabra Santa

Faculda de de Ciências Médicas da Santa Casa de São Paulo

Departamento de Medicina Social

Rua Cesário Motta Jr., 120 - Santa Cecília

05409 - São Paulo - SP

33. Sonra Latoz das Chagas e Silva

Fundação Saúde Caetano Munhoz da Rocha

Rua Engo Rebouças, 1707

80000 - Curitiba - PR

34. Erci José Soar Filho

Fundação Saúde Caetano Munhoz Rocha

$\mathrm{CDRH}$

Praça Ouvidor Pardinho, s/no

80000 - Curitiba - PR

35. Darli Antonio Soares

Universidade Estadual de Londrina

Hospital Universitário

Av. Robert Koch, s/no

86100 - Londrina - PR

36. Ana Misako Yendo Ito

Universidade Estadual de Londrina

Hospital Universitário

Av. Rober to Koch, s/n?

86100 - Londrina - PR

37. Severno Ramos de Lima

Universidade Federal da Paraíba

Rua Dom Bosco, 121 - Cristo Redentor

(endereço residencial)

58000 - João Pessoa - PB

38. Marilisa Berti de Azevedo Barros

Departamento de Medicina Preventiva e Social

UNICAMP

Rua João de Souza Campos, 34

13100 - Campinas - SP

39. Maria das Graças Soares Costa

Secretaria de Saúde

Av. Duque de Caxias, 948

01000 - São Paulo - SP

40. Terezinha Faria da Costa

Caciernos de Saúde Pública, RJ, 3 (1):81-97, Jan/fev, 1987 
NAMPS - Alagoas

Praça dos Palm ares - Centro

57000 - Maceió - AL

41. Vera Lúcia Kodjaoglanian

Delegacia Federal de Saúde/MS

Rua João Pessoa, 333 - Vila Planalto

79100 - Campo Grande - MS

42. Sebastião de Moraes

Secretaria de Saúde de Itu

Rua Barāo de Itaim, 140

13300 - Itu - SP

43. Maurício Roberto Campelo de Macedo

Universidade Federal do Rio Grande do Norte

Departamento de Saúde Coletiva e Nutrição

Rua Marechal Cordeiro de Farias, s/nọ - Petrópolis

59000 - Natal - RN

44. Celina Maria Turchi Matelli

Universidade Federal de Goiás (IPTESP)

Praça Universitária, $s / n$ ?

74000 - Goiânia - GO

45. Dorothy Silva Lima

Departamento de Medicina Preventiva

Un iversidade Federal de Goiás

Praça Universitária, $s / n$ ?

74000 - Goiânia - GO

46. Divaneide Ferreira de Sousa

Secretaria de Saúde Pública do Rio Grande do Norte

Av. Junqueira Aires, 488 - Centro

59000 - Natal - RN

47. Roberto Batista Pedrosa

Departamento de Medicina Preventiva e Saúde Coletiva

IPTSP

74000 - Goiânia - GO

48. Sérgio Dantas Chagas

Universidade Federal do Rio Grande do Norte

Rua Cordeiro de Farias, s/no - Petrópolis

59000 - Natal - RN

49. Maria do Carmo Lessa Guimarães

Universidade Federal da Bahia

Rua J - Q. 16 - Lote 22 - Edifício Paikerê

40000 - Salvador - BA

50. Zorilda Andrade Vaz da Silva

Secretaria de Saúde da Bahia

Rua Amazonas, 14 - Apto 703 - Pituba

(Endereço residencial)

40000 - Salvador - BA

51. Mércia Costa Jones

Rua das Margaridas - Edifício Cerejeira/24.

Pituba (End. res.)

40000 - Salvador - BA

52. Leonora Rodrigues de Castro

Faculdade de Gências da Saúde

Rua Dr. Martin Santana, 1053 
69000 - Manaus - AM

53. Neide Paula Miranda de Carvalho

Secretaria de Saúde do Amazonas

Estrada do Contorno $\mathrm{s} / \mathrm{n}$ ?

69000 - Manaus - AM

54. Walny Bittencourt Ferreira

Secretaria de Saúde do Pará

Rua Presidentc Pernam buco, 489

66000 - Belém - PA

55. Victor Hugo Corrêa

Secretaria de Saú de do Pará

Rua Presidente Pernambuco, 489

66000 - Belém - PA 